\title{
The Role of Language in Ethnic Identity Measurement: A Multitrait-multimethod Approach to Construct Validation
}

\author{
MICHEL LAROCHE \\ Concordia University \\ FRANK PONS \\ Université Laval \\ MARIE-ODILE RICHARD \\ University of Montreal
}

\section{Please direct correspondence to:}

\author{
Michel Laroche, Royal Bank Distinguished Professor of Marketing \\ Department of Marketing, John Molson School of Business \\ Concordia University, 1455 de Maisonneuve West \\ Montreal, Quebec, Canada H3G 1M8
}

Tel: (514) 848-2424 ext. 2942, Fax: (514) 848-4576

Email: laroche@jmsb.concordia.ca

Acknowledgments: The authors thank Isabelle Miodek for her valuable assistance and gratefully acknowledge the financial support of the Fonds Québécois de la recherche sur la société et la culture, Quebec (Canada). We also acknowledge the helpful comments of the editor and two anonymous reviewers.

\begin{abstract}
In this study we focus on the role of language use in measuring ethnic identity. We demonstrate the construct validity of a three dimensional measure, and examines potential biases introduced by using alternative methods such as constant sum scale. Our findings support the distinction between the three sub-dimensions of language use (English use in family, in media consumption, while shopping). We found evidence of discriminant validity. By using three approaches to construct validity, our support for discriminant validity strengthens the discriminant and convergent properties of the instrument. Our findings concerning method effects are less obvious. If the traditional multitrait-multimethod approach indicates no method effects, more stringent approaches (ANOVA and (UA) indicate the presence of limited method effects. We provide implications regarding measurement of ethnic identity.
\end{abstract}

Key words: ethnic identity, language use, measurement, multitrait-multimethod

THE ETHNIC IDENTITY CONCEPT has been of interest to multicultural researchers in many fields such as cultural adaptation studies (Anderson \& Frideres, 1981; Berry, 1997; Roosens, 1989), counseling psychology (Cokley, 2007; Phinney \& Ong, 2007) and marketing (Laroche, Kim, \& Tomiuk, 1998; Laroche, Kim, Tomiuk, \& Belisle, 2005). Cokley (2007, p. 224) states that "ethnic and racial identity are among the most researched topics in the multicultural counseling literature. The popularity of these constructs, combined with ongoing controversies surrounding their measurement, warrants a critical examination by scholars in the field."

Ethnic identity is defined as the retention of features from an individual's culture of origin (Keefe \& Padilla, 1987; Laroche, Kim, \& Tomiuk, 1998). In fact, this retention of the culture of origin is expressed through attitudes, values or behaviors (Masuda, Matsumoto, \& Meredith, 1970; White \& Burke, 1985). This definition of ethnic identity broadens the restrictive sense given to the term by several studies that only focus on the ethnicity of origin or the country of origin (Daghfous \& d'Astous, 1991; Kim, Laroche, \& Tomiuk, 2004). Most researchers 
agree that ethnicity stems from the fact that certain individuals 'belong to' or 'identify with' certain ethnic groups (Phinney, 1990; Phinney \& Ong, 2007). Laroche et al. (1998, p. 419) conceptualize ethnicity (ethnic identity) as “...the character or quality encompassing several cultural indicators which are used to assign people to groupings."

This stream of research also underlines the complex and multidimensional nature of the concept and the importance of self-reported measures to capture the dimensions forming ethnic identity (Lee \& Yoo, 2004).

Phinney (1990) proposes that "ethnic involvement," which encompasses language, friends networks, religious affiliation, participation in structured ethnic social groups (e.g., sports), political ideology, area of residence and other miscellaneous activities (e.g., consumption of media, foods, and songs) be the most widely used indicator of multigroup ethnic identity. Indeed, she develops, then revises the Multigroup Ethnic Identity Measure (MEIM-R; Phinney \& Ong, 2007) that incorporates six self-report items of attitudinal and behavioral features of ethnic identity and broadly measures ethnic identity across two major dimensions (i.e., exploration and commitment). Researchers in psychology acknowledge it as the most popular tool to capture ethnic identity (Lee, Flabo, Doh, \& Park, 2001; Mack et al., 1997) and many researchers adopted it in studying relationships of ethnic identity with numerous psychological adjustment and well-being measures, including self-esteem, depression, anxiety, social connectedness, and loneliness (Lee, 2002; Roberts et al., 1999).

\section{Ethnic Identity and Ethnic Groups}

Anderson (1980) addresses the complexity of ethnic identity, questions the multigroup approach and argues that dimensions that form ethnic identity are unique to the cultural group that is studied. In this stream of research, each ethnic group can be defined by its own dimensions of ethnic identity. Ethnic identity researchers on specific cultural groups have used and developed specific scales such as the Suinn-Lew Asian Self-Identity Acculturation Scale (SL-ASIA; Suinn, Rickard-Figueroa, Lew, \& Vigil, 1987), the Internal-External Ethnic Identity Measure (Kwan, 2000) or the General Ethnicity Questionnaire (Tsai, Ying, \& Lee, 2000) for Chinese Americans. Other researchers use the measurement of racial identity status in the context of racial minority-majority group dynamics (e.g., Alvarez \& Helms, 2001). These approaches to the study of ethnic identity are important contributions to the field and are preferable when studying ethnic outcomes that are specific to each group (e.g., role conflicts within Korean families). Many dimensions for specific cultural groups have been presented in the literature as components of ethnic identity (Aboud \& Christian, 1979; Laroche et al., 1998). These dimensions usually vary widely depending 
on the origin of the ethnic group at stake. Indeed, specific features of a given ethnic group form the ethnic identity of one of its member.

Education and socialization. Education and socialization play an important role in the maintenance of ethnic groups and the development of ethnic identity dimensions. Though in the past "ethnic education" was almost exclusively informal, more organized classes in religion, language, and tradition appeared as learning in the home cannot always be assumed, especially for immigrants (Cohen, 2004). Ethnic boundaries seem constructed mainly by an individual's self-identification with what is taught in social networks (Stodolska \& Yi, 2003). Among these key dimensions, language is described as the most salient aspect of ethnic identity in particular in studies on Chinese-Canadians (Kim et al., 2004), Franco-Americans in Northern Maine (Giles, Taylor, Lambert, \& Albert, 1976), French-Canadians (Laroche, Kim, Hui, \& Joy, 1996; Taylor, Bassili, \& Aboud, 1973), Italian- and Greek-Canadians (Laroche, Kim, \& Hui, 1997; Laroche et al., 2005), and South Africans (Korf \& Malan, 2002). Other researchers also consider language as a secondary factor and rather focus on religion or involvement within ethnic activities (e.g., organizations, cultural traditions; Dashefsky \& Shapiro, 1974; Theodoratus, 1971). This is the case in studies dealing with Baltic States and Belarus in particular (Ioffe, 2003). In this study, the author shows how Belarusian ethnic identity is difficult to define, how the native Belarusian language still does not rally citizens and ethnic identity is rather built on different features such as religion.

Role of language in forming ethnic identity. In the majority of studies dealing with ethnic identity, language along with religious affiliation, participation in clubs and food preferences are the most widely accepted and used dimensions of ethnic identity (Laroche et al., 1998). Following this line of research, we deal with the role of language in forming ethnic identity and more specifically how using the English language contributes to the definition of the Anglo-Canadian ethnic identity. We particularly investigate measurement issues related to the use of this language in measuring ethnic identity.

This issue is particularly interesting in the wake of controversies regarding language-related measures in the U.S. census. The main criticisms concern some ethnic groups such as American Indians (Liebler, 2004). Most researchers on self-reported ethnic identity use Likert-type scales to assess how often the respondents' language(s) of reference is(are) used in different situations (work/leisure/family), leaving open the possibility of having multiple languages of reference. This approach may lead to several ethnic classifications for the same individual and then to difficulties in interpreting results regarding ethnic representation within a group (Phinney, 1996; Ponterotto, 
Gretchen, Utsey, Stracuzzi, \& Saya, 2003). Concerns have been expressed following the 2000 U.S. census where individuals could classify themselves in up to six different ethnic groups. Some ethnic group associations in the U.S. were afraid of the potential dilution of their members and of losing their overall representation and power of negotiation for a fair representation in the American society (Gonzalez, 1999). In order to limit classification options and therefore to push respondents to define a dominant ethnic group to identity with, some researchers use alternative methods of measurement such as a limited number of choices or the use of a constant sum scale (e.g., 100 points to allocate among alternatives) to measure ethnic identity dimensions and language use in particular.

Our main objective is to refine the dimensions of language use to allow for the measurement of the ethnic identity of English-speaking respondents in a major Canadian city. Furthermore, the use of two methods, namely the Likert-type scale and the constant sum scale to assess each of these dimensions should help determine to what extent any variation in the measurement is due to random measurement error or systematic sources of error, i.e., a bias due to the method in particular (Bagozzi \& Yi, 1991). The use of construct validation will allow to (a) clearly define language use as a dimension of ethnic identity by using two different methods, and $(b)$ see how well these two methods measure this construct. The following two hypotheses are formulated as"

Hypothesis $1\left(\mathrm{H}_{1}\right)$ : The language use dimension of ethnic identity of English-Canadians is composed of several sub-dimensions which show convergent and discriminant validities.

$\mathrm{H}_{2}$ : The methods of Likert-type scale and constant sum scale have equivalent measurement properties.

\section{Method}

\section{Sample and Data Collection}

The target population consists of English Canadians residing in a major metropolitan area. We obtain a random sample from eighteen randomly selected census tracts located within the city and its surrounding areas. A sample of at least 150 to 200 usable questionnaires is deemed appropriate for this research to give it a respectable measure of validity and reliability (i.e., 10 respondents per item; Carmines \& MacIver, 1981).

Based on past experience, we expect a usable return rate of $30 \%$. Within each census tracts, we establish a distribution quota proportionally to the population of the census tract. We select streets at random in each census tract and try to survey as many households on these streets as possible until we reach this quota. We choose residential districts with detached or semi-detached dwellings for ease of access, whereas we avoid apartment 
districts for lack of access. We administer the questionnaires door to door and give qualifying respondents willing to participate a questionnaire and a prepaid envelope for mailing it back once completed. We distribute a total of 898 questionnaires and receive 228 usable questionnaires for a $25.4 \%$ response rate.

\section{Measures}

In the cover letter, we ask respondents to willingly participate in a study on Canadian lifestyles.

Participation is fully voluntary without any deception. We receive approval of the university ethics committee prior to distributing the questionnaires.

The questionnaire is structured in three parts. In the first one, we ask questions pertaining to lifestyles, and included, 12 items measuring the extent of English use in different contexts (e.g., family, work, shopping, and media; Table 1a and Table 1b). We measure these items on a 9-point Likert-type scale $(1=$ disagree strongly to $9=$ agree strongly). In the second part we ask the degree to which an individual uses English compared to French in daily activities. We operationalize these measures as the percentage of English and French language use for different media (e.g., TV, radio, and newspapers) as well as for interactions with other people (e.g., spouse/husband, children, co-workers, and friends). We ask respondents to distribute 100 points between English and French for each situation. This procedure, called a constant sum scale, allows evaluating the relative use of the languages made by the respondents. In fact, in the first two parts we propose two different methods to measure dimensions of language in forming English ethnic identity. These sub-dimensions are respectively intra social network use of English (i.e., spouse/husband, children, relatives, friends, and co-workers), use of English for media consumption (i.e., TV, radio, and newspaper) and use of English for everyday activities such as shopping. As the use of English in family is an important aspect of ethnic identity (particularly the use of English between spouses), we decide to include only respondents with a spouse. Therefore the final data set went from 228 respondents to 180 , still above the threshold recommended to perform confirmatory factor analysis (i.e., 10 respondents per item; Carmines \& MacIver, 1981). In the third part we assess the demographic characteristics of the sample. Consistent with previous studies, we ask information such as age, gender, education and family income.

\section{[Table 1a and Table $1 b$ about here]}

\section{Results}

\section{Sample Demographics}

In terms of age, respondents between 40 and 49 years old account for 30 percent, those between 20 and 29 
for 27 percent, those below 30 for 7 percent, those between 50 and 59 for 19 percent, and those above 60 for 17 percent. In terms of family income, the sample is slightly skewed toward the mid to high levels, with 44 percent over $\$ 80 \mathrm{k}, 33$ percent between $\$ 50 \mathrm{k}$ and $\$ 80 \mathrm{k}$, and 23 percent below $\$ 50 \mathrm{k}$. In terms of education, 26 percent have a high school degree, 25 percent a community college degree, 33 percent an undergraduate degree and 16 percent a graduate degree. Finally, females account for $57 \%$ of the sample.

\section{Measure Development}

Each respondent provides one Likert-type measure of the perceived extent of English used in different contexts as well as a percentage measure of English used in the same contexts. The percentage measurement is a constant sum scale with ratio-scale properties. We can use it as a second measurement method for the language dimension of ethnic identity. Moreover, given the close nature of scales content, respondents' assessment of their use of English using the Likert-type scale should provide as reliable measures as the ones using constant sum scales. Table 1 provides the means and standard deviations of all measures.

The advantage of a second method is that it enables us to use multitrait-multimethod approaches to evaluate the validity of the scale (Bagozzi, 1993; Campbell \& Fiske, 1959). For the data analysis we follow the process proposed by Kim and Lee (1997):

1. We use a measure purification process to obtain a set of reliable items common to the two methods (Likert-type and constant sum) for each construct. This is based on inter item correlation as well as factor analysis. The contents of the measures (items and corresponding dimensions) resulting from each method must be equivalent.

2. We conduct a confirmatory factor analyses of the common sets of items obtained from each method.

3. We conduct a confirmatory factor analyses to the equivalence of the factor structure for both methods.

4. We conduct a multitrait-multimethod analysis to test the construct validity of the language dimensions of ethnic identity.

Measure Purification. First, we use exploratory factor analyses (EFA) to refine the instrument and obtain a set of items equivalent between the two methods. With the EFA we can examine the patterns in the data, which might suggest congruence between empirical results and the underlying theory (Churchill, 1979; Stevens, 1986). Although EFA has been criticized if used by itself (Gould, 1981), we use it prior to a confirmatory factor analysis (CFA) to obtain the factorial structure that will be tested in the CFA. We can identify items with poor psychometric properties 
and delete them from the instrument (Campbell, 1960; Churchill, 1979). Roehrich (1994) suggests that identification of the optimal factorial structure be an iterative process in which successive EFAs are performed after each item's elimination until all items and dimensions have reasonable psychometric properties and equivalence between the methods used.

After the first EFA, we examine the qualities of the items and apply selection rules. These rules are mainly based on the variance shared between an item and its construct. Rivard and Huff (1988) suggest a minimum value of 0.5 for this shared variance (i.e., loadings above 0.7). Kline (1998) considers factor loadings as high if they are greater than 0.6 (the sign being irrelevant). However, these strict rules can be accommodated somewhat on the condition that an item only loads on the dimension it is intended to measure and not on others (Pedhazur \& Pedhazur-Schmelkin, 1991). In addition, inter-item correlation among items measuring the same dimension should be high (Roehrich, 1994) and reliability coefficients for each dimension in each method should be above the Nunnally's (1967) criterion with Cronbach alphas all above 0.7. Before examining, the factorial structure and the reliability of each dimension, we perform several successive EFAs. In fact, after the deletion of any item with poor psychometric properties, we perform a new EFA.

In Tables 2 and 3, we present the results of the first EFAs respectively performed on the 12 Likert-type scale items and the 12 constant sum items. We use an Oblimin rotation. In the case of two or more factors extracted, with rotation we can better interpret the coefficients matrix (Norusis, 1988). We use Oblimin rotation because conceptually first order factors should be correlated. For both EFAs, we extract 3 factors using the criterion of eigenvalues greater than one. Respectively $57 \%$ and $66 \%$ of the variance are explained for the Likert-type method and the constant sum method. We include only loadings above the 0.25 threshold. We remove items with weak loadings on the dimension they are supposed to measure as well as those with cross-loading above 0.25 on more than one factor. We also perform a verification of the theoretical meaning attached to the withdrawal of these items. Therefore, we remove items LIKE22, LIKE28 and LIKE49 in the Likert-type method and LFRIEND1, LSCHOOL1 and LWORK1 in the constant sum method with cross-loadings on other dimensions (see Tables 2 and 3 in bold). The deleted items are common to both methods. Following Kim and Lee's (1997) approach, our deletion of these items indicates how well measurement items relate to the hypothesised dimensions. Only three items remain in the first dimension; they are all related to the use of English within the family, reducing the social network by excluding items about schoolmates and friends. This dimension is renamed intra-family use of English. The two items 
remaining in the third dimension are directly related to the amount of English used for shopping activities.

[Table 2 about here]

[Table 3 about here]

We perform a second EFA on the new sets of items ( 9 for each method). In Tables 4 and 5, we present the results, showing only cross-loadings above 0.25 . Following the Kaiser-Guttman's criterion, we extract three factors for each method. They explain $65 \%$ of the variance for the Likert-type method and $71 \%$ for the constant sum method. All items in the three-factor solution have loadings above or close to 0.7 , and none has significant crossloadings. Thus, the iterative measure purification process results in 9 items and the factor patterns are highly similar across the two methods. Reliabilities for each dimension and each method show very satisfying results (Nunnally, 1967) with Cronbach alphas all above 0.7. Intra-family use of English, use of English for media consumption and use of English for shopping activities have respectively Cronbach alphas of 0.74, 0.84 and 0.70 for the Likert-type method; $0.83,0.78$ and 0.71 for the constant sum method.

[Table 4 about here]

[Table 5 about here]

Based on these results, the 9 English use items for each method are good indicators of their respective dimensions. In Table 6 we present these items, as well as their related dimensions.

[Table 6 about here]

Confirmatory Factor Analysis. We subject the purified 9-item measurement model for each of the method to confirmatory factor analyses using the EQS software. In Figure 1 we present the model. For both the Likert-type scale method and the constant sum scale one, the three-factor measurement model indicate a satisfactory fit according to the $\chi^{2} /$ degree of freedom criterion (Anderson \& Gerbing, 1988; Bollen, 1989; Browne \& Kudeck, 1989; Tanaka, 1993) and the Comparative Fit Index (CFI), which measures the relative improvement of the hypothesised model over a null model while taking into account relatively small sample sizes (Bentler, 1989). The acceptance criterion for the $\chi^{2} / \mathrm{df}$ ranges between 5 to 6 for the least stringent methods (Wheaton, Muthèn, Alwin, \& Summers, 1977), and 4 to 3 for stricter approaches (Carmines \& MacIver, 1981). The cut-off value of CFIs suggested for an adequate fit is 0.90 (Bentler $\&$ Bonett, 1980). The $\chi^{2} / \mathrm{df}$ values are respectively $2.17\left(\chi^{2}(24, N=\right.$ $202)=52.1, p<.01)$ and $2.06\left(\chi^{2}(24, N=210)=49.5, p<.01\right)$ for the constant sum scale and the Likert-type scale measures; whereas the CFIs are 0.94 and 0.94 respectively. All factor loadings for the two methods are significant $(t$ 
$>1.96)$.

Therefore, hypothesis H1 is supported for both the constant sum scale and the Likert-type scale methods.

\section{[Figure 1 about here]}

Multisample Confirmatory Factor Analysis. We put the three-factor measurement model, confirmed separately for each method, to a more stringent test of structural equivalence across the two methods. Considering each method as a specific sample, we perform a set of multisample confirmatory factor analyses to test the following models, which hypothesize the invariance of various parameters (Joreskog \& Sorbom, 1989; Kim \& Lee, 1997):

Model 1: For each method, there are three correlated factors with the same pattern factor (Figure 1). That is all parameters are estimated freely without any restriction in each method.

Model 2: Factor loadings are invariant in both cases. They are constrained equal but all other parameters are freely estimated (error variances and factor covariance).

Model 3: Error variances are invariant in both cases. They are constrained equal but all other parameters are freely estimated (factor loadings and factor covariances).

Model 4: Factor covariances are invariant in both cases. They are constrained equal but all other parameters are freely estimated (error variances and factor loadings).

Model 5: Models 2 and 3. Factor loadings and error variances are invariant in both cases. They are respectively constrained equal but factor covariances are freely estimated

Model 6: Models 2 and 4. Factor loadings and factor covariances are invariant in both cases. They are respectively constrained equal but error variances are freely estimated.

Model 7: Models 2, 3 and 4. Factor loadings, error variances and factor covariances are invariant in both cases. They are respectively constrained equal.

In Table 7 we present the fit results. Model 1, for which we hypothesise three correlated common factors for each method is the least restrictive of the seven. All others impose different set of constraints on the parameters of this baseline model. Therefore, Models 2-7 are nested in Model 1 (Kim \& Lee, 1997). Using $\chi^{2}$ difference tests, Models 2, 3 and 5 are not significantly different from the baseline model $\left(\chi^{2}(5, N=198)=2.6, p>.05 ; \chi^{2}(10, N=\right.$ $198)=7.2, p>.05$ and $\left.\chi^{2}(15, N=198)=9.1, p>.05\right)$. Among these three models, Model 5 is nested in both Model 2 and Model 3. Moreover, additional $\chi^{2}$ difference tests between Model 5 and respectively Models 2 and 3indicate no significant difference $\left(\chi^{2}(10, N=198)=6.6, p>.05 ; \chi^{2}(5, N=198)=1.90, p>.05\right)$. Consequently, Model 5, 
which hypothesizes invariant factor loadings as well as error variances, is indicative of the common measurement properties for the two methods. This result indicates that the 9 items have at least the same dimensionality (i.e., factor patterns), factor structure (i.e., factor loadings), but probably not the same factor covariances across the two methods. Partial invariance is confirmed by the results of the Lagrange Multiplier Test on the constraints that we could release during the confirmatory analysis. This simultaneous test of the constraints allows identification of nonequivalent parameters (Byrne, 1994). In this case, three constraints should be released and they all deal with the invariance of factor covariances. Therefore, one can conclude to partial invariance of the measurement instrument.

[Table 7 about here]

\section{Construct Validation of the Language Dimension in Assessing Ethnic Identity}

Having confirmed the equivalent use of language structure for both methods, the next task is to conduct construct validation on the three dimensional structure. Construct validity is the extent to which a construct achieves empirical and theoretical meaning (Steenkamp \& Van Tripp, 1991). Lord and Novick (1968) posited that "for scientific purposes, the most important characteristic of a test is its construct validity." Construct validity is made operational through the assessment of convergent and discriminant validities. In the assessment of construct validity, Campbell and Fiske (1959) propose to examine measures of multiple traits each measured with multiple methods, namely the multitrait-multimethod approach. Three different multitrait-multimethod approaches are found in the literature.

The multitrait-multimethod matrix (Campbell \& Fiske, 1959). We assess convergent and discriminant validity of language use dimensions by looking at the monotrait-heteromethod, heterotrait-monomethod and heterotraitheteromethod values in the multitrait-multimethod correlation matrix. Items measuring the same trait but using different methods (monotrait-heteromethod) should have a higher correlation than items measuring different traits using the same method (heterotrait-monomethod). We should find the smallest correlations among items measuring different traits using different methods (heterotrait-heteromethod). In Table 8 we present the multitrait-multimethod correlation matrix. Convergent validity is assessed through the analysis of the validity diagonals. They should be large and statistically significant. The average of these coefficients (bold in the table) is 0.57 . All the coefficients are statistically significant $(p<.0001)$ suggesting convergent validity for the ethnic identity measurement. 
A comparison between monotrait-heteromethod correlations and heterotrait-heteromethod correlations indicates that the monotrait-heteromethod values are higher in all cases except one. Moreover, the validity coefficient (average of monotrait-heteromethod correlations) is larger (0.57) than all the heterotrait-heteromethod correlations. Furthermore, the monotrait-heteromethod correlations exceed in all but one case the heterotraitmonomethod correlations. This is a more stringent support to the establishment of discriminant validity for language use and indirectly for ethnic identity. It also suggests relatively limited method effects in the measurement of language use using the Likert-type scale or the constant sum scale. However, the multitrait-multimethod matrix approach is very subjective as a statistical test of significance is not provided (Humbert \& Baker, 1979; Widaman, 1985). Also, precise estimates of trait or method variances are not given. Alternative techniques have been used to overcome these problems.

The analysis of variance approach (Kavanagh et al., 1981). By using measures from respondents for each level of trait and method, we estimate three orthogonal sources of variation. We treat respondents as random effects, traits and methods as fixed effects. With this analysis we measure three effects, namely: $(a)$ the main effect of subjects to assess convergent validity, if there are significant differences between subjects for measures averaged across traits and methods, $(b)$ the subject by trait interaction effect which tests if differences between subjects depend on traits and provides an overall estimate of discriminant validity, and $(c)$ the subject by method interaction effect which estimates method biases (Schmitt \& Stults, 1986).

In Table 9 we present the results. There is a statistically significant main effect of subjects indicating convergent validity, $F(1,179)=1.18, p<.05$. The subject by trait interaction is also statistically significant, $F(2,358)$ $=1.322, p<.05$. This shows that traits have a differential validity, meaning that individuals are ranked differently for the different traits measuring language use. Finally, the subject by method interaction is statistically significant, $F(1,179)=2.15, p<.001$. This suggests that the method used to measure language use traits may be responsible for differentiations in the results among subjects. These method effects introduce a systematic source of undesirable variance (Marsh, 1989). If the ANOVA approach seems more appropriate than the multitrait-multimethod matrix in the sense that it gives more systematic criteria evaluation, it has been criticized for not accounting for measurement error (Schmitt, Coyle, \& Saari, 1977), and using a different convergent validity definition than Campbell and Fiske (Marsh \& Hocevar, 1983). Therefore, we use a third approach to assess construct validity. 
The general confirmatory factor analysis applied to MTMM data (Widaman, 1985). The first step is to assess the magnitudes of traits and methods effects through a hierarchical approach (Widaman, 1985) by using a set of successive confirmatory factor analyses (MTMM) as suggested by Bagozzi (1993) and Kim and Lee (1997). We use the three dimensions of language use as "traits" and Likert-type measures as well as constant sum ones as the two "methods." The Likert-type measures and the constant sums are averaged separately over the set of items measuring each respective dimension. Each trait had only two indicators (one from the Likert-type measure average on this dimension and the other from the constant sum average on the same dimension). Figure 2 presents the multitraitmultimethod model. This model (M4) is the last of the four nested models to estimate and it includes both traits and methods and their respective inter-correlations but none between traits and methods. The first model (M1) is a null model in which only random error is present. Model 2 (M2) is a method-only model in which we only estimate method variances. Model 3 (M3) is a trait-only model in which we only include traits variances.

\section{[Figure 2 about here]}

In Table 10 we present the respective estimations of Models 1-4. If we do not encounter special problems for the first three models, we cannot estimate Model 4 because of an under-identification problem (i.e., 21 data points for 22 free parameters). This problem is typical of MTMM with less than 3 traits and 3 methods (Kenny, 1979). Several solutions have been offered to resolve these identification issues. The Correlated Uniqueness Approach (CUA, Marsh, 1989) is the best alternative proposed. In this approach, we do not represent method effects by separate method factors but rather by correlated errors between the same method measures. Using this approach, we respecify Model 4. Unfortunately, the improvement in the number of parameters to estimate is not sufficient as only two methods are used. The model is just specified (i.e., same number of parameters to estimate as data points), leading to poorly defined solutions. We make one last attempt to use CUA by keeping this time method factors but replacing traits factors by correlated error terms between the same traits measures. This method is similar to the usual CUA but the interpretation has to be different as the focus switches from method to traits and vice-versa. In Figure 3 we present the Correlated Uniqueness model.

\section{[Table 10 about here]}

\section{[Figure 3 about here]}

With the null method we obtain a poor fit, whereas we obtain significant fits for the traits only and CUA, with a better one for the CUA. The successive comparisons of the models are always significant and suggest that 
with the CUA we have the best fit to the data. Using the model with method effects and correlated errors to represent the traits effects we perform much better than the other ones. Consequently, it appears that if traits effects are superior to method effects, it would be important to consider that both method and trait effects have to be accounted for to explain the variance in language use measurement.

In Table 11 we present the factor loadings on the two method factors resulting from the CUA (M4) specification. The loadings are all statistically significant. However, the error terms are quite important and the percent of variance extracted is small. Consequently, even if the loadings are significant, there are signs that the method effects are indeed quite limited and the significant correlation between the two method factors $(0.81)$ also argues for limited method effects.

\section{[Table 11 about here]}

We study the traits effects through the correlation of error terms related to respective traits and provided in the CUA results. To have traits effects, we should have important and significant correlations between these error terms. The correlations between errors of items measuring the same trait through different methods are all significant ranging from 0.63 for 'English use in family' to 0.34 for 'English use in media consumption'. The correlation between errors for measurement of 'English use while shopping' between constant sum and Likert-type is 0.49 . These results support differentiation between the measurement of traits related to the language use and the definition of ethnic identity while maintaining consistency through different types of measures.

Therefore, hypothesis $\mathrm{H} 2$ is largely supported across the three different multitrait-multimethod approaches.

\section{Discussion}

The purpose of this study is twofold. First, by focusing on the role of language use in the measurement of ethnic identity, we develop a three-dimensional scale using standard psychometric tools, and demonstrate the construct validity of this scale. The multidimensional nature of ethnic identity is widely acknowledged and researchers have tried to develop several types of instruments ranging from language to food consumption or religion (Phinney \& Ong, 2007; Saloutos, 1980), but few specifically worked on measurement issues and many authors insisted on the need for valid and reliable instruments of ethnic identity and acculturation (Cockley, 2007; Laroche et al., 1998). In this study, we focus only on language use in different situations and show that each dimension of ethnic identity may have distinct sub-dimensions that lead to a better understanding of ethnic identity 
in general. Second, we try to examine the potential biases introduced by the use of alternative types of measurements in surveys such as constant sum scale items.

Overall, we provide support for the conceptual distinction between the three sub-dimensions of language use, namely English use in family, in media consumption and while shopping. With the findings regarding evidence of discriminant validity we support this distinction. Moreover, with the use of three different approaches to analyse construct validity and the constant support for discriminant validity across the three methods we provide stronger evidence of the discriminant and convergent validities of this scale.

The findings concerning method effects are less obvious. If the traditional multitrait-multimethod approach (Campbell \& Fiske, 1959) indicates that there are no method effects, more stringent tests such as analysis of variance, MTMM and the correlated uniqueness approach indicate the presence of method effects. However, these effects seem limited and the two methods are convergent on several aspects. Furthermore, with the lack of significance of methods-only effects, we support its limited impact on the overall measurement process. The analysis of factor loadings for each method can also indicate the quality of measurement for each individual trait. For instance, the measure of 'English use in media consumption' seems better when using constant sum scale whereas in the case of 'English use in family' the use of a Likert-type scale seems more appropriate. We need more research to confirm and refine these findings and also to extend possible use of these alternate types of measurement. In the future, researchers should also try to investigate other dimensions of ethnic identity.

Our support for the construct validity of the tri-dimensional scale of language use in assessing ethnic identity strengthens previous findings in ethnic identity research. However, with the very limited impact of measurement methods and more specifically of constant sum scale on language use measurement, we open interesting avenues for researchers and practitioners who may choose to limit alternative responses offered to respondents or at least require from them a ranking of their choices. If the limited influence on construct validity is confirmed in future research, the possibility of using alternative measurement tools without sacrificing quality of or altering answers may be the most important findings of this study. Since ethnic identity has been used in a variety of domains such as counseling psychology, education, socialization and adaptation, health behaviour (e.g., depression, anxiety, social connectedness, and loneliness), it is important to have a valid scale of language use dimensions of ethnic identity to include in larger models which might be developed in the future to study these important outcomes. We propose a short and valid scale which can be easily used by researchers. 


\section{Limitations and Future Research}

Finally, this study has some limitations. First, the sample is not fully representative of the target population, as some segments were excluded for lack of access. In the future researchers should try to reach these segments, maybe by accessing appropriate shopping malls. Second, we exclude single respondents (a small group in this sample) because the measure of intra-family language use does not yet apply to them. In the future, researchers should try other means to include them in the measure, such as use of English with parents and close friends, or through other dimensions of ethnic identity. Third, researchers can only apply this measure to ethnic groups where English use is an issue; however a great majority of ethnic identity and acculturation researchers deal with immigrants groups which have to learn English in their adopted countries, such as: Italians, Greeks, North Africans, Lebanese, East Asians, Chinese, and Koreans. So using measures of language use in different contexts to assess ethnic identity and acculturation is most appropriate for the vast majority of immigrant groups.

Future researchers should apply the approach developed in this paper to other ethnic groups for whom language use is an important dimension of their ethnic identity, such as Italians, Greeks, Chinese, Koreans, Lebanese and North Africans. Also researchers might develop other dimensions of ethnic identity and acculturation and apply them in various cross-cultural research programs, such as persuasion research (Michon \& Chebat, 2004; Rosenbaum \& Montoya, 2007), food choices and food preference research (Laroche et al., 2005) and internet surfing research (Richard, 2005).

\section{AUTHOR NOTES}

Michel Laroche is the Royal Bank Distinguished Professor of Marketing at Concordia University in Montreal (Canada). His main research interests are in the areas of consumer behavior, culture, services marketing, internet and persuasion research. Frank Pons is Associate Professor of Marketing at Laval University, Québec City (Canada). His main research interests are in the areas of consumer behavior and services marketing with an emphasis on crosscultural issues and sports marketing. Marie-Odile Richard is a Post-Doctoral Fellow in neuromarketing at the University of Montreal. Her main research interests are in the areas of internet research, services marketing and neuroscience applications to marketing. 


\section{REFERENCES}

Aboud, F. E., \& Christian, J. (1979). Development of ethnic identity. In L. Eckensberger, Y. Poortinga, \& W. J. Lonner (Eds.). Cross-cultural contributions to psychology. Lisse, Holland: Swets \& Zeitlinger.

Alvarez, A. N., \& Helms, J. E. (2001). Racial identity and reflected appraisals as influences on Asian Americans' racial adjustment. Cultural Diversity and Ethnic Minority Psychology, 7(3), 217-231. DOI: 10.1037//10999809.7.3.217

Anderson, A. B. (1980). The survival of ethno-linguistic minorities: Canadian and comparative research. In H. Giles, \& B. St Jacques (Eds.). Language and ethnic interaction (pp. 67-79). Oxford: Pergamon.

Anderson, A. B., \& Frideres, J. S. (1981). Ethnicity in Canada: Theoretical perspectives. Toronto, CN: Buttersworth.

Anderson J., \& Gerbing D. (1988). Structural equation modeling in practice: A review and recommended two-step approach. Psychological Bulletin, 103(3), 411-423.

Bagozzi R. P. (1993). Assessing construct validity in personality research: Applications to measures of self-esteem. Journal of Research in Personality, 27(1), 49-87.

Bagozzi, R. P., \& Yi, Y. (1991). Multitrait-multimethod matrices in consumer research. Journal of Consumer Research, 17(4), 426-439.

Bentler, P. (1989). EQS: Structural equations program manual. Los Angeles, CA: BMDP Statistical Software.

Bentler P., \& Bonett, D. (1980). Significance test and goodness of fit in the analysis of covariance structures. Psychological Bulletin, 88(3), 588-606. DOI: 1981-06898-001.

Berry, J. W. (1997), Immigration, acculturation, and adaptation. Applied Psychology: An International Review, 46, 1, 5-34. DOI:10.1111/j.1464-0597.1997.tb01087.x

Bollen, K. (1989). Structural equations with latent variables. New York: John Wiley.

Browne, M.W., \& Cudeck, R. (1989). Single sample cross validation indices for covariance structures. Multivariate Behavioral Research, 24(9), 445-455.

DOI: $10.1207 / \mathrm{s} 15327906 \mathrm{mbr} 2404 \_4$

Byrne, B. (1994). Structural equation modeling with EQS and EQS/Windows. Thousand Oaks, CA: Sage.

Campbell, D. (1960). Recommendations for APA test standards regarding trait, construct or discriminant validity. American Psychologist, 15(8), 546-553.

Campbell, D., \& Fiske, D. (1959). Convergent and discriminant validation by the multitrait-multimethod matrix. Psychological Bulletin, 56(2), 81-105.

Carmines, E., \& McIver, J. (1981). Analyzing models with unobserved variables: Analysis of covariance structures. In G. Bohrnstedt \& E. Borgatta (Eds.). Social Measurement: Current issues (pp. 65-115). Newbury Park, CA: Sage.

Churchill, G. (1979). A paradigm for constructing better measures of marketing concepts. Journal of Marketing Research, 16(1), 64-73.

Cohen, E. H. (2004). Components and symbols of ethnic identity: A case study in informal education and identity formation in diaspora. Applied Psychology: An International Review, 53(1), 87-112. 
DOI:10.1111/j.1464-0597.2004.00162.x

Cokley, K. (2007), Critical issues in the measurement of ethnic and racial identity: A referendum on the state of the field. Journal of Counseling Psychology, 54(3), 224-234. doi:10.1037/0022-0167.54.3.224

Daghfous, N., \& d'Astous, A. (1991). The effects of acculturation and length of residency on consumption-related behaviors and orientations of Arab-Muslim immigrants. In T. Schellinck (Ed.). 1991 Conference proceedings (pp. 91-101). Niagara Falls, CN: Administrative Sciences Association of Canada.

Dashefsky, A., \& H. Shapiro (1974). Ethnic identification among American Jews: Socialization and social structure. Lexington, MA: Lexington Books.

Giles, H. W., Taylor, D. M., Lambert, W. E., \& Albert, G. (1976). Dimensions of ethnic identity: An example from Northern Maine. Journal of Social Psychology, 100(1), 11-19.

Gonzalez, R. (1999). Census 2000: What about race? Wall Street Journal. January 21, 12.

Gould, S. (1981). The mismeasure of man. New York: Norton.

Ioffe, G. (2003). Understanding Belarus: Belarusian identity. Europe-Asia Studies, 55(8), 1241-1272. DOI: $10.1080 / 0966813032000141114$

Jöreskog, K., \& Sörbom, D. (1989). LISREL 7: A guide to the program and applications. Chicago, IL: SPSS.

Kavanagh, M. J., MacKinney, A. C., \& Wolins, L. (1971). Issues in managerial performance: Multitraitmultimethod analyses of ratings. Psychological Bulletin, 75(1), 34-49.

Keefe, S. E., \& Padilla, A. M. (1987). Chicano ethnicity. Albuquerque, NM: University of New Mexico Press.

Kenny, D. A. (1979). Correlation and causality. New York: Wiley.

Kim, C., Laroche, M., \& Tomiuk, M. (2004). The Chinese in Canada: A study in ethnic change with emphasis on gender roles. Journal of Social Psychology, 144(1), 5-29. DOI: 10.3200/SOCP.144.1.5-29

Kim, C., \& Lee, H. (1997). Development of family triadic measures for children's purchase influence. Journal of Marketing Research, 34(3), 307-321.

Kline, R. B. (1998). Principles and practice of structural equation modeling. New York: The Guilford Press.

Korf, L., \& Malan, J. (2002). Threat to ethnic identity: The experience of White Afrikaans-speaking participants in postapartheid South Africa. Journal of Social Psychology, 142(2), 149-170.

Kwan, K. L. K. (2000). The internal-external ethnic identity measure: Factor-analytic structures based on a sample of Chinese-Americans. Educational and Psychological Measurement, 60(1), 142-152.

DOI: $10.1177 / 00131640021970321$

Laroche, M., Kim, C., \& Hui, M. (1997). A comparative investigation of dimensional structures of acculturation for Italian- and Greek-Canadians. Journal of Social Psychology, 137(3), 317-331.

Laroche, M., Kim, C., Hui, M., \& Joy, A. (1996). An empirical study of multidimensional ethnic change: The case of the French Canadians in Quebec. Journal of Cross-Cultural Psychology, 27(1), 114-131. DOI: $10.1177 / 0022022196271008$

Laroche, M., Kim, C., \& Tomiuk, M. (1998). A test of nonlinear relationship between linguistic acculturation and ethnic identification. Journal of Cross-Cultural Psychology, 29(3), 418-433. 
DOI: $10.1177 / 0022022198293003$

Laroche, M., Kim, C., Tomiuk, M., \& Belisle, D. (2005). Similarities in Italian and Greek multidimensional ethnic identity: Some implications for food consumption. Canadian Journal of Administrative Sciences, 21(4), 143167.

Lee, J. S. (2002). The Korean language in America: The role of cultural identity in heritage language learning. Language, Culture and Curriculum, 15(2), 117-33.

Lee, R. M., Falbo, T., Doh, H. S., \& Park, S. Y. (2001). The Korean diasporic experience: Measuring ethnic identity in the United States and China. Cultural Diversity and Ethnic Minority Psychology, 7(3), 207-216.

Lee, R. M., \& Yoo, H. C. (2004). Structure and measurement of ethnic identity for Asian American college students. Journal of Counseling Psychology, 51(2), 263-269. DOI: 10.1037/0022-0167.51.2.263

Liebler, C. A. (2004). Ties on the fringes of identity. Social Science Research, 33(4), 702-723. DOI:10.1016/j.ssresearch.2003.10.002

Lord, F., \& Novick, M. (1968). Statistical theories of mental test scores. Reading, MA: Addison-Wesley.

Mack, D. E., Tucker, T. W., Archuleta, R., DeGroot, G., Hernandez, A. A., \& Oh Cha, S. (1997). Interethnic relations on campus: Can't we all get along? Journal of Multicultural Counseling and Development, 25(4), 256-268.

Marsh, H. W. (1989). Confirmatory factor analysis of multitrait-multimethod data: Many problems and a few solutions. Applied Psychological Measurement, 13(4), 335-361. DOI: 10.1177/014662168901300402

Marsh, H. W., \& Hocevar, D. (1983). Confirmatory factor analysis of multitrait-multimethod matrices. Journal of Educational Measurement, 20(3), 231-248. doi:10.1111/j.1745-3984.1983.tb00202.x

Masuda, M., Matsumoto, G. H., \& Meredith, G. M. (1970). Ethnic identity in three generations of Japanese Americans. Journal of Social Psychology, 81(2), 199-207.

Michon, R., \& Chebat, J. C. (2004). Cross-cultural mall shopping values and habitats: A comparison between English- and French-speaking Canadians. Journal of Business Research, 57(8), 883-892. DOI: S0148-2963(02)00291-6

Norusis, M. J. (1988). SPSS-X advanced statistics guide. Chicago, IL: SPSS

Nunnally, J. (1967). Psychometric theory. New York: McGraw-Hill.

Pedhazur, E., \& Pedhazur-Schmelkin, L. (1991). Measurement, design and analysis: An integrated approach. Hillsdale, NJ: Lawrence Erlbaum.

Phinney, J. S. (1990). Ethnic identity in adolescents and adults: Review and research. Psychological Bulletin, $108(3), 499-514$.

Phinney, J. S. (1992). The multigroup ethnic identity measure: A new scale for use with diverse groups. Journal of Adolescent Research, 7(2), 156-176. DOI: 10.1177/074355489272003

Phinney, J. S. (1996). When we talk about American ethnic groups, what do we mean? American Psychologist, 51(9), 918-927.

Phinney, J. S. \& Ong, A. D. (2007), Conceptualization and measurement of ethnic identity: current status and future directions, Journal of Counseling Psychology, 54(3), 271-281. doi:10.1037/0022-0167.54.3.271 
Ponterotto, J. G., Gretchen, D., Utsey, S. O., Stracuzzi, T., \& Saya, R. (2003). The multigroup ethnic identity measure: Psychometric review and further validity testing. Educational and Psychological Measurement, 63(3), 502-515. DOI: 10.1177/0013164403063003010

Richard, M. O. (2005). Modeling the impact of internet atmospherics on surfer behavior. Journal of Business Research, 58(12), 1632-1642. DOI:10.1016/j.jbusres.2004.07.009

Rivard, S., \& Huff, S. (1988). Factors of success for end user computing. Communications of the ACM, 31(5), 552561. doi.acm.org/10.1145/42411.42418

Roberts, R., Phinney, J., Masse, L., Chen, Y., Roberts, C., \& Romero, A. (1999). The structure of ethnic identity in young adolescents from diverse ethnocultural groups. Journal of Early Adolescence, 19(3), 301-322. DOI: $10.1177 / 0272431699019003001$

Roehrich, G. (1994). Innovativité hédoniste et sociale: Proposition d'une échelle de mesure. Recherche et Applications en Marketing, 9(2), 19-42.

Roosens, E. E. (1989). Creating ethnicity, London: Sage.

Rosenbaum, M. S., \& Montoya, D. Y. (2007). Am I welcome here? Exploring how ethnic consumers assess their place identity. Journal of Business Research, 60(3), 206-214. DOI:10.1016/j.jbusres.2006.09.026

Saloutos, T. (1980). Greeks. In S. Thermstrom (Ed.). Harvard encyclopedia of American ethnic groups (pp. 430440), Cambridge, MA: Harvard University Press.

Schmitt, N., \& Stults, D. M. (1986). Methodological review: Analysis of multitrait-multimethod matrices. Applied Psychological Measurement, 10(1), 1-22. DOI: 10.1177/014662168601000101

Schmitt, N., Coyle, B. W., \& Saari, B. B. (1977). A review and critique of analyses of multitrait-multimethod matrices. Multivariate Behavioral Research, 12(4), 447-478.

Steenkamp, J.-B. E. M., \& van Trijp, H. C. M. (1991). The use of LISREL in validating marketing constructs. International Journal of Research in Marketing, 8(3), 283-299.

Stevens, J. (1986). Applied multivariate statistics for the social sciences. Hillsdale, NJ: Erlbaum.

Stodolska, M. \& Yi., J. (2003). Impacts of immigration on ethnic identity and leisure behavior of adolescent immigrants from Korea, Mexico, and Poland. Journal of Leisure Research, 35(1), 49-79.

Suinn, R. M., Rickard-Figueroa, K., Lew, S., \& Vigil, P. (1987). The Suinn-Lew Asian self-identity acculturation scale: An initial report. Educational and Psychological Measurement, 47(2), 401-407. DOI: $10.1177 / 0013164487472012$

Tanaka, J. (1993). Multifaceted conceptions of fit in structural equation models. Testing structural equation models (pp. 10-39). Newbury Park, CA: Sage.

Taylor, D. M., Bassili, J. N., \& Aboud, F. E. (1973). Dimensions of ethnic identity: An example from Quebec. Journal of Social Psychology, 89(2), 185-192.

Theodoratus, R. J. (1971). A Greek community in America: Tacoma, Washington. Sacramento State College: Sacramento Anthropological Society.

Tsai, J. L., Ying, Y.-W., \& Lee, P. A. (2000). The meaning of 'being Chinese' and 'being American': Variation among Chinese American young adults. Journal of Cross-Cultural Psychology, 31(3), 302-332.

DOI: $10.1177 / 0022022100031003002$ 
Wheaton, B., Muthèn, B., Alwin, D., \& Summers, G. (1977). Assessing reliability and stability in panel models. In D. R. Heise (Ed.), Sociological Methodology (pp. 84-136), San Francisco, CA: Jossey-Bass.

White, C., \& Burke, P. (1987). Ethnic role identity among White and Black college students: An interactionist approach. Sociological Perspectives, 30(3), 310-331.

Widaman, K. F. (1985). Hierarchically nested covariance structure models for multitrait-multimethod data. Applied Psychological Measurement, 9(1), 1-26. DOI: 10.1177/014662168500900101 